\title{
The prediction of recurrence in meningiomas
}

\author{
M. RUFUS CROMPTON AND PETER C. GAUTIER-SMITH \\ From the Atkinson Morley's Hospital, Wimbledon, and the National Hospital for Nervous Diseases, \\ Queen Square
}

The purpose of this investigation was to examine a large series of surgically removed meningiomas and correlate the microscopic pathological features found in them with recurrence-free survival of the patient or recurrence of the tumour. It has never been clear what information the pathologist can give to the clinician in a patient who has had a meningioma removed - that is, apart from informing him that it is indeed a meningioma. Simpson (1957) summarized the literature on the recurrence of meningiomas and presented his own findings. However, these were mainly based on surgical findings and macroscopic pathology. A detailed study of the significance of the histology of these tumours has not appeared in the medical literature. It would be desirable to be able to give the clinician some pretty firm opinion as to the likelihood of recurrence. Such information together with the patient's age and general health might render radiotherapy unnecessary in a number of cases.

It was not the purpose of this investigation to provide a detailed account of the pathology of meningiomas, as such accounts are available elsewhere (Cushing and Eisenhardt, 1938; Courville, 1945; Russell and Rubinstein, 1959).

The 181 tumours examined were all from patients treated by or under the care of Mr. Wylie McKissock between 1936 and the end of 1966 . All but a very few were admitted either to Atkinson Morley's Hospital, Wimbledon, or the National Hospital, Queen Square, London. All are parasagittal or falcine tumours, as these are of especial clinical interest and will be the subject of a future publication. It is thought that most of the information obtained will apply to meningiomas at other sites.

The likelihood of recurrence with various tumour types and pathological features was expressed as a 'recurrence ratio'. This was reached by dividing the number of cases surviving $5 \frac{1}{2}$ years or more, free from recurrence, by the number with recurrences. Thirty six patients had recurrences of their tumours between three months and 14 years of operation. The majority of recurrences occurred around $5 \frac{1}{2}$ years after operation. The first part of the paper is $\frac{\bar{O}}{\overline{0}}$ concerned with recurrence in the 141 patients in $\overparen{\odot}$ whom the surgeon felt sure of complete removal of the tumour, and then there is a comment on the ${ }^{\mathrm{s}}$ 40 in whom he felt that the tumour was incompletely $\vec{O}$ removed.

The histological classification of tumours used $\vec{\omega}$ was that of Courville (1945) which was adopted by Russell and Rubinstein (1959). This divides the -0 tumours into syncytial, transitional, fibroblastic, $\underset{\omega}{\omega}$ and angioblastic forms. All but three of the tumours fitted into one of these categories. One of these thrge was a frank sarcoma, probably a fibrosarcom. The other two were identical in appearance; thes were made up of cells having a primitive or embro $\vec{T}$ onic appearance suggestive of fibroblasts in tissu룽 culture. They had an open-work arrangement wigh spaces between the branching processes of the cex (Fig. 1). There was abundant reticulin of angiês: blastic type associated with the individual cets (Fig. 2) and the mitotic rate was high. They almost. certainly represent a variant of the angioblastic tumours. Many specimens were of tumours which had regions showing the features of two or more of the above categories, and in these cases the $\stackrel{\square}{\not}$ tumour was classified on the dominant pattern. $\stackrel{\unrhd}{\circledR}$ The most frequent examples were syncytial tu- $\overrightarrow{\vec{B}}$ mours with transitional regions. The periphery of $\frac{\circ}{3}$ tumours was often syncytial and the centre tran- $?$ sitional. There were transitional tumours witho fibroblastic regions and vice versa, but very seldom were syncytial tumours combined with fibroblastic tumours. This all suggested a phylogenetically in- - creased degree of differentiation of the tumours 3 from syncytial through transitional to fibroblastic type. Angioblastic tumours sometimes contained transitional, non-vasoformative regions, suggesting that they are true meningiomas developed from the versatile, multi-potential arachnoidal or menin- $-\frac{7}{0}$ gothelial cell and not developed from the endothelium of meningeal blood vessels. In no patient in whom N the tumour had recurred, and both tumours had N been examined histologically, had the tumour N 


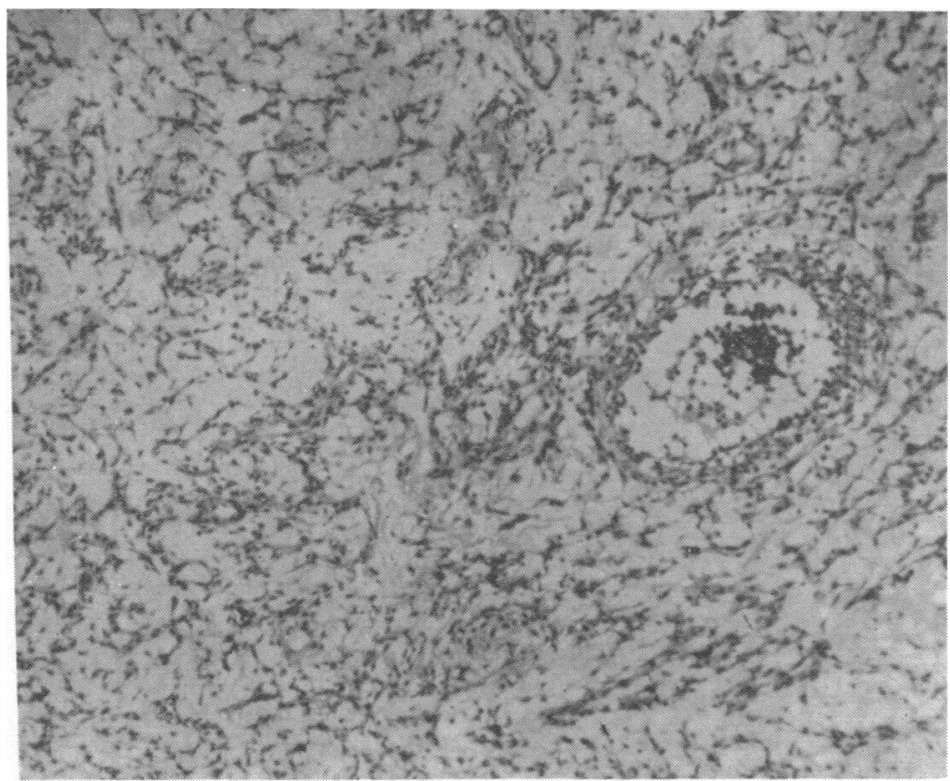

FIG. 1. An openwork or embryonic type of meningioma showing the lacy, tissue-culture-like appearance.

Haematoxylin and eosin, $\times 90$.

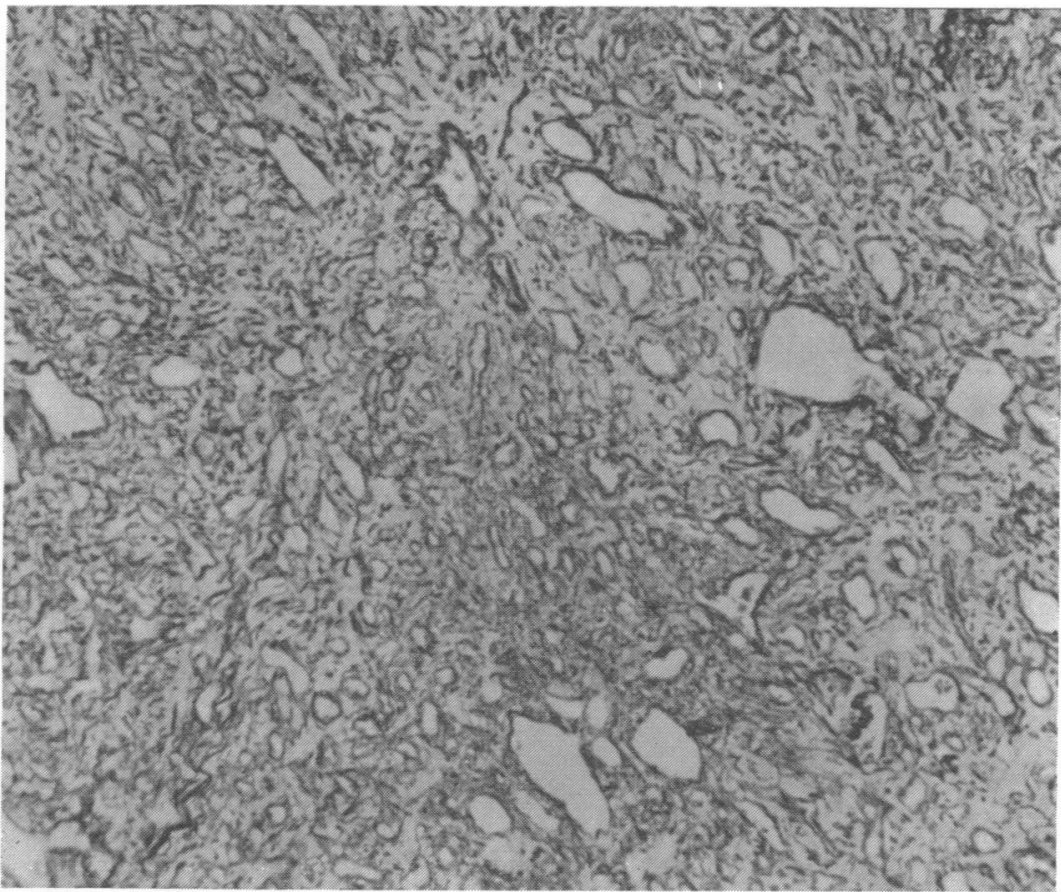

FIG. 2. An openwork or embryonic type of meningioma showing the rich angioblastic type of reticulin. Reticulin silver impregnation, $\times 85$. 
changed its basic type, though it may have appeared to have a different rate of growth as suggested by more, or fewer, mitotic figures.

The tumours from 181 patients were examined. Table 1 shows the number in each of the categories described. This merely confirms the impression of the majority of pathologists of the predominance of the transitional meningioma, the infrequency of the angioblastic tumours and the rarity of frank sarcomas and other variants.

TABLE 1

THE NUMBER OF DIFFERENT TUMOUR TYPES

\begin{tabular}{lcc}
\hline & Number & Percentage of total (\%) \\
\cline { 2 - 3 } Syncytial & 38 & 21 \\
Transitional & 96 & 53 \\
Fibroblastic & 35 & 19 \\
Angioblastic & 9 & 5 \\
Sarcoma & 1 & 2 \\
Embryonic or open-work & 2 & \\
\hline
\end{tabular}

The recurrence ratio after complete removal for the tumour types were syncytial 13/7(1.9), transitional $41 / 12(3.4)$ and fibroblastic 20/1 (20). The angioblastic tumours had four long survivals and no recurrences. The one sarcoma survived over $5 \frac{1}{2}$ years without recurrence. Thus, the fibrous meningiomas, probably the best differentiated, were less likely to recur than the other common varieties.

There are certain additional histological features which have frequently been described in meningiomas and with which most pathologists are familiar. The frequency of some of these features in the four main categories of the present series is shown in Table II. The features noted were psammoma bodies (P), either hyaline or hyaline and calcified; foamy cells $(F)$ or cells with foamy or clear cytoplasm containing sudanophilic lipid; grossly pleomorphic nuclei $(\mathrm{N})$ with great variation of size, shape, and density of chromatin; vacuolated nuclei (V) consisting of clear, colourless nuclear vacuoles; nuclear inclusions (I) which are eosinophilic and probably represent invaginations of cytoplasm; small cysts (C) as opposed to massive central cystic breakdown; adipose tissue (A) or fully differentiated fat cells as opposed to foamy cells and bone (B) as opposed to mere calcification.

Some points that emerge from this table are: (1) Psammoma bodies occurred in about one-fifth of transitional tumours, and, if they occurred in syncytial or fibroblastic types, it was in a transitional region. (2) Foamy cells appeared in about $10 \%$ of both syncytial and transitional tumours. They did not appear in fibroblastic tumours, nor did nuclear pleomorphism. (3) Vacuolated nuclei were present
TABLE 2

THE DISTRIBUTION OF SOME HISTOLOGICAL FEATURES

\begin{tabular}{ccccc}
\hline & Syncytial & Transitional & Fibroblastic & Angioblastic \\
\hline Number & & & & \\
examined & 38 & 96 & 35 & 9 \\
P & 1 & 21 & 4 & \\
F & 3 & 9 & & \\
N & 6 & 7 & 2 & \\
V & 4 & 10 & & \\
I & 2 & 5 & & \\
C & 1 & 3 & & \\
A & & 3 & & \\
B & & 1 & &
\end{tabular}

$\mathbf{P}=$ Psammoma bodies, $\mathbf{F}=$ Foamy cells, $\mathbf{N}=$ Grossly pleomorphic nuclei, $\mathbf{V}=$ Vacuolated nuclei, $\mathbf{I}=$ Nuclear inclusions, $\mathrm{C}=$ Small cysts, $\mathbf{A}=$ Adipose tissue, $\mathbf{B}=$ Bone formation.

in about $10 \%$ of syncytial, transitional and fibroblastic tumours. (4) Nuclear inclusions were not seen in fibroblastic meningiomas. (5) Gross nuclear pleomorphism did not appear in fibroblastic tumours. (6) Small cysts did not appear in fibrous meningiomas. (7) Fat occurred only in transitional tumours.

Most of these figures are too small for useful analysis, but no relationship could be found between any of these features, and future recurrence of the tumour.

\section{MITOTIC FIGURES}

Mitotic figures, indicating the organization anfe pairing of genes before cell division, are apparent as a rearrangement of nuclear chromatin. In manye tumours throughout the body they indicate rapidity of growth, and in others they also indicate malignancy. We feel that in meningiomas they represent rapid growth and not malignancy. The word sarcoma should not be used for a meningioma of one of the well-known types if it contains mitotic figures, but should be reserved for variants resembling fibrosarcomas as seen elsewhere in the body. Mitotic figures are often difficult to distinguish from pyknotic nuclei in meningiomas, and should always be checked under a high power objective. The rod-like mitoses seen in the bipolar cells of fibroblastic meningiomas lie across the cell at right angles to its long axis. An attempt was made to assess the frequency of mitotic figures in the various meningioma types as shown in Table 3. Mitoses were recorded if at least three unequivocal figures were seen in tumour cells and not stromal or vascular endothelial cells. No attempt was made to quantitate the frequency of mitosis. Mitoses occurred in $\mathbf{8 1}$ $(45 \%)$ of the 181 meningiomas examined. This is at variance with the belief that they are exceptional in these tumours. All three atypical tumours- 
TABLE 3

THE DISTRIBUTION OF MTTOTIC FIGURES

\begin{tabular}{lcccc}
\hline & Syncytial & Transitional & Fibroblastic & Angioblastic \\
\cline { 2 - 5 } $\begin{array}{l}\text { Total number } \\
\text { examined }\end{array}$ & 38 & 96 & 35 & 9 \\
$\begin{array}{l}\text { Number with } \\
\text { mitoses }\end{array}$ & 23 & 37 & 13 & 6 \\
$\begin{array}{l}\text { Percentages of } \\
\text { total (\%) }\end{array}$ & 60 & 38 & 38 & 66 \\
\hline
\end{tabular}

namely, the sarcoma and the two 'embryonic' or 'open-work' variants-contained abundant mitoses. It can be seen from Table 3 that there was a considerably higher mitotic rate in syncytial tumours than in transitional or fibroblastic ones. It is interesting that sometimes the peripheral and possibly most rapidly growing portion of some predominantly transitional tumours is syncytial in type, and also that mitotic activity is more prominent at the periphery of most meningiomas, whether this is at the boundary with brain, dura, or bone. The angioblastic tumours contained many mitoses. This appears to be at variance with their lack of recurrence in this series. However, their numbers are too small for any definite conclusions to be drawn.

The recurrence ratio in the meningiomas of this series, completely removed and containing mitotic figures, was $26 / 14(1 \cdot 85)$, whereas that for tumours in which no mitotic figures were seen was $55 / 6(9 \cdot 1)$. Thus mitotic figures are apparently a factor in assessing likelihood of recurrence in these meningiomas.

\section{NECROSIS}

This was defined to exclude the presence of regions of foamy or clear cells and the presence of small cysts, but to include massive breakdown of the centre of a large tumour with cyst formation and also to include small, focal regions of necrosis throughout the tumour (Figs. 3 and 4). In rare instances, such as one of the two 'embryonic' tumours, there was thrombosis of vessels within

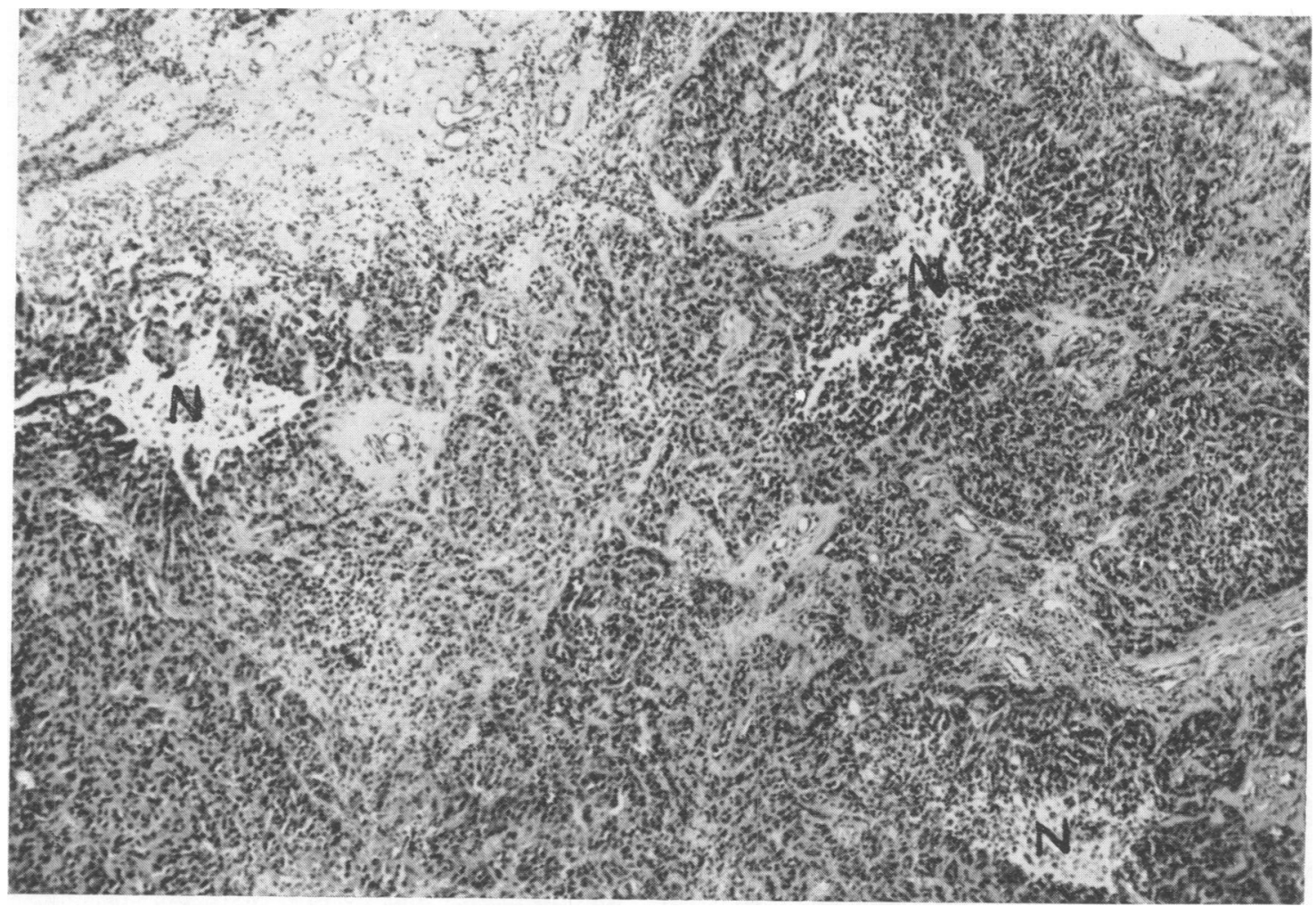

FIG. 3. A low power view of three regions of focal necrosis $(N)$ in a meningioma. Haematoxylin and eosin, $\times 45$. 


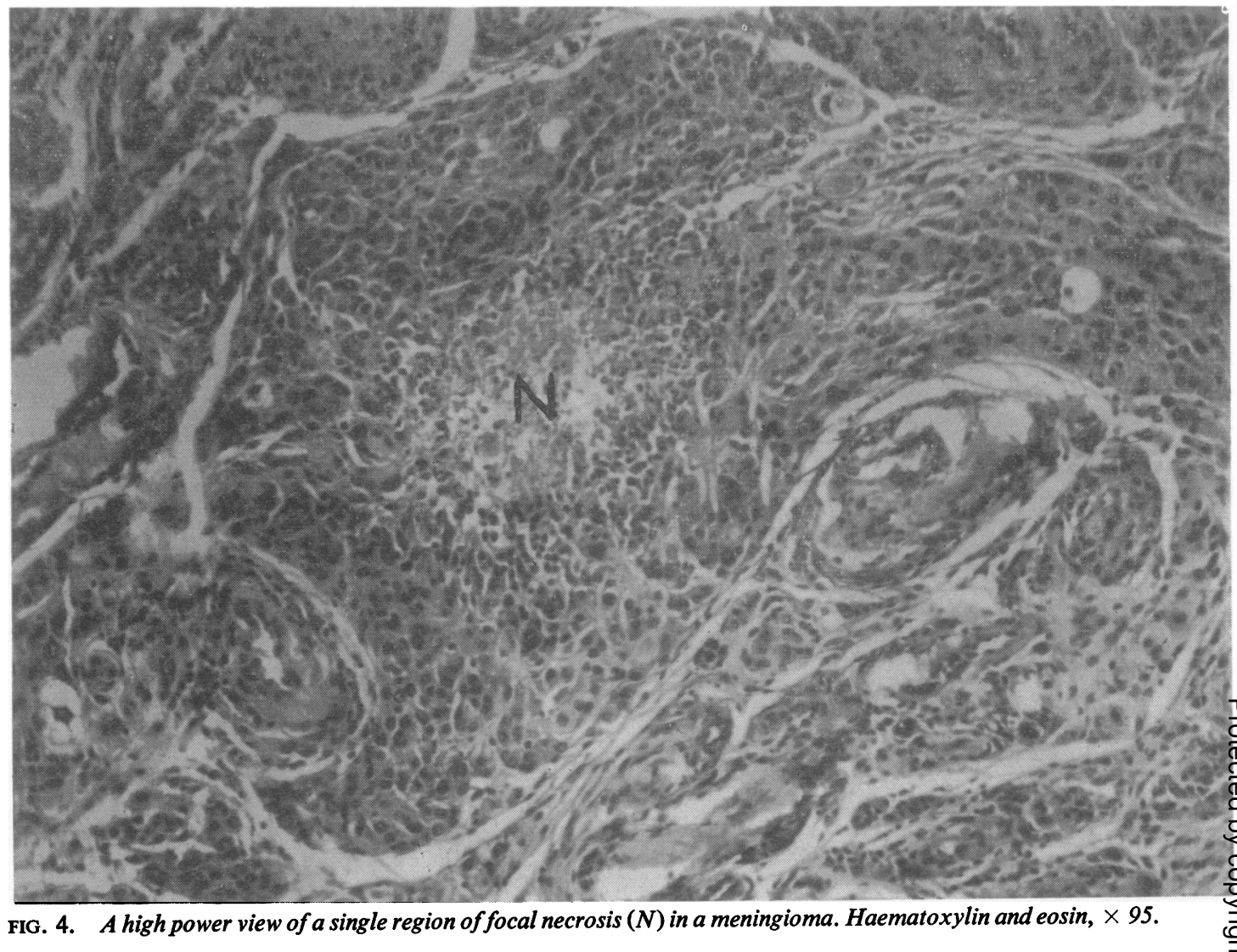

FIG. 4. A high power view of a single region of focal necrosis $(N)$ in a meningioma. Haematoxylin and eosin, $\times 95$.

the tumour and resultant inarction of tumour substance. However, obvious vessel changes did not appear to be the basis of tumour necrosis in the great majority of cases.

Fifty $(27 \cdot 5 \%)$ out of 181 tumours contained necrotic regions. In 29 of them it was central necrosis and in 29 it was focal necrosis. In eight cases both types of necrosis were present. Table 4 shows the incidence of necrosis in the main tumour types. It was also present in the one sarcoma and one of the two 'embryonic' tumours which are not included in this table. In each type, focal and central necrosis occurred with about equal frequency. As with mitotic figures, the syncytial tumours contained most regions of necrosis, and again the few angioblastic tumours frequently contained necrotic areas. This again was at variance with their freedom from recurrence.

The recurrence ratio for the tumours completely removed and showing focal necrosis was $8 / 8(1.0)$ and for those with no focal necrosis it was 78/12 $(6 \cdot 5)$. This suggests that, like mitoses, regions of focal necrosis are indicative of likely recurrence in
TABLE 4

THE INCIDENCE OF NECROSIS IN THE TUMOUR TYPES

\begin{tabular}{|c|c|c|c|c|}
\hline & Syncytial & Transitional & Fibroblastic & Angioblastic \\
\hline \multirow{4}{*}{$\begin{array}{l}\text { Total number } \\
\text { examined } \\
\text { Number with } \\
\text { necrosis } \\
\text { Percentage of } \\
\text { the total (\%) } \\
\text { (Number with } \\
\text { focal necrosis) }\end{array}$} & 38 & 96 & 35 & 9 \\
\hline & 14 & 24 & 5 & 5 \\
\hline & 37 & 25 & 15 & 55 \\
\hline & (9) & (13) & (2) & (3) \\
\hline
\end{tabular}

these meningiomas. Central necrosis is probably an expression of the size of a tumour and does not have the same significance as focal necrosis with regard to tumour recurrence.

\section{RELATION OF NECROSIS TO MITOTIC FIGURES}

Both these features occurred regularly in syncytial and angioblastic meningiomas. In the 181 cases, of mitotic figures and necrosis were present together $N$ in 32, and in 26 of these it was focal necrosis. There $\underset{\omega}{\mathcal{N}}$ 
appears to be a relationship between mitoses and focal necrosis, both probably indicating rapid growth. Table 5 shows the incidence of this combination in the different tumour types. It was most frequent in the angioblastic and least frequent in the fibroblastic types, probably because necrosis is uncommon in fibroblastic tumours.

The recurrence ratio for tumours with both focal necrosis and mitotic figures was 7/5 (1.4) which lies between that for focal necrosis $(1 \cdot 0)$ and mitotic figures (1·85).

TABLE 5

THE INCIDENCE OF COMBINED FOCAL NECROSIS AND MITOSES

\begin{tabular}{lcccc}
\hline & Syncytial & Transitional & Fibroblastic & Angioblastic \\
\cline { 2 - 5 } $\begin{array}{l}\text { Total number } \\
\text { examined }\end{array}$ & 38 & 96 & 35 & 9 \\
$\begin{array}{l}\text { Number with } \\
\text { combination }\end{array}$ & 9 & 16 & 2 & 5 \\
$\begin{array}{l}\text { Percentage of } \\
\text { the total (\%) }\end{array}$ & 15 & 19 & 5 & 55 \\
\hline
\end{tabular}

CEREBRAL INFILTRATION

In some tumours stubby little processes or fingers of tumour cells extend for varying distances into the cerebral cortex. The appearance was reminiscent of the downward extension of a basal cell carcinoma into the dermis (Fig. 5). This usually resulted in a thin layer of cortex being removed with the tumour, and one wonders if small nests of tumour cells were not left behind in the tumour bed.

In both infiltrating and non-infiltrating tumours there was occasionally a prominent astrocytic reaction and proliferation at the tumour-brain boundary (Fig. 6). This was referred to as the 'glial barrier', and assessed in relation to cerebral infiltration and mitoses. The presence or absence of cerebral infiltration could be estimated histologically in 70 cases. Infiltration of the brain was present in $17(24 \%)$ of these, of which $10(59 \%)$ had a 'glial barrier', whereas this was present in only nine $(17 \%)$ of the 53 tumours that did not infiltrate the cortex. Thus there appeared to be

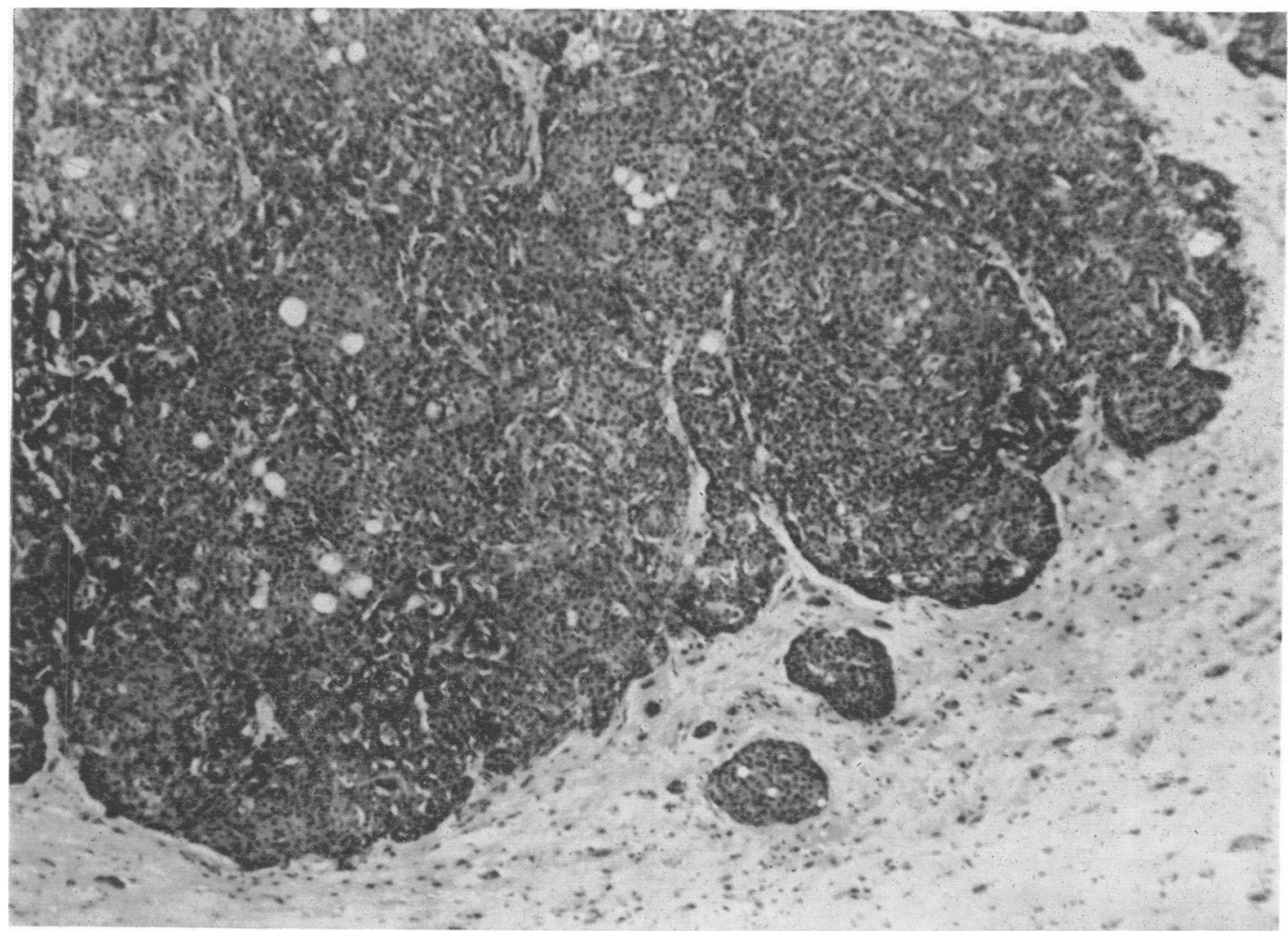

FIG. 5. Short processes of t umour above, infiltrating into the cerebral cortex below. Haematoxylin and eosin, $\times 60$. 


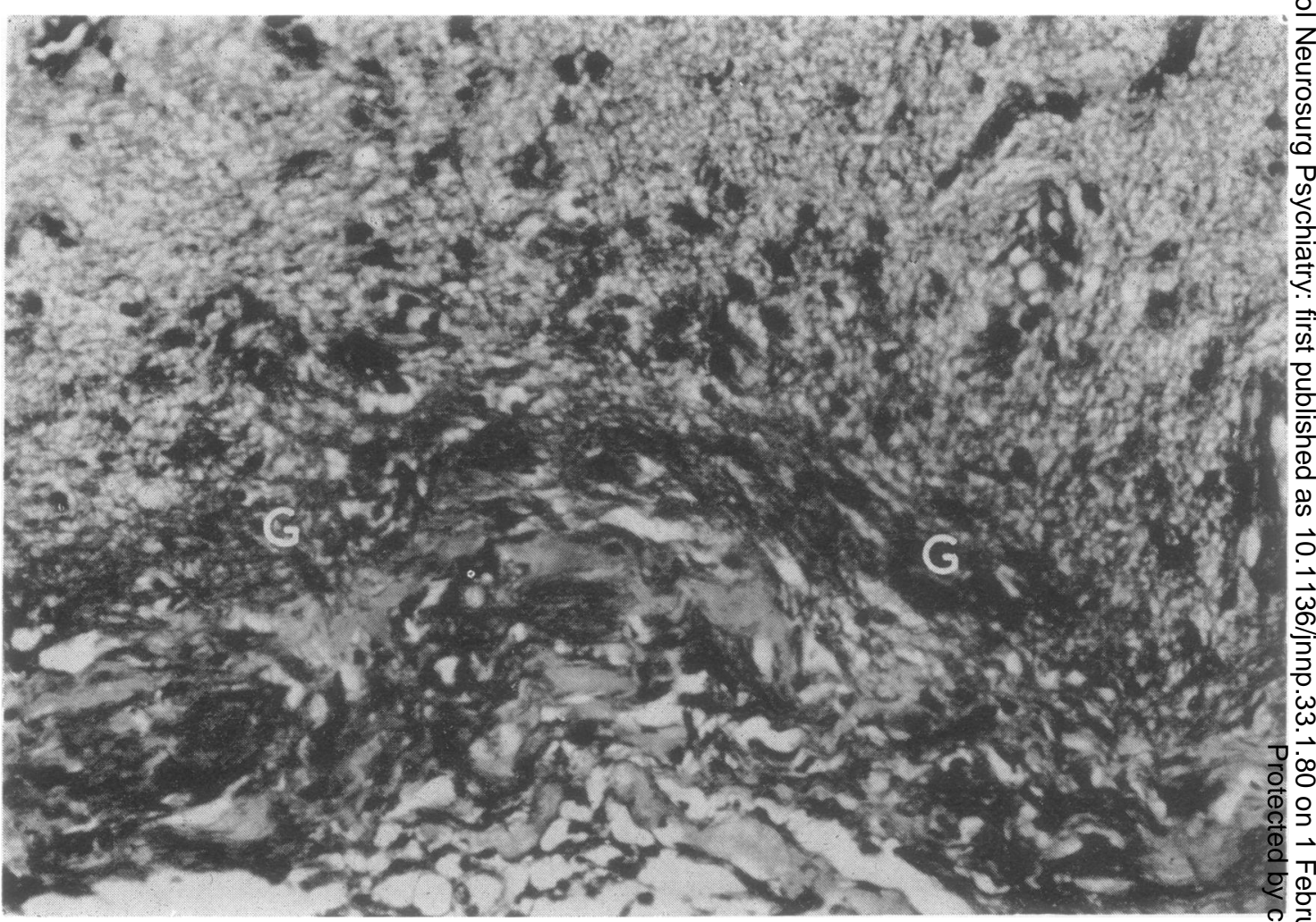

FIG. 6. A dense band of darkly staining fibrous astrocytes constituting the glial barrier (G) between the cerebral cor above and the tumour margin below. Phosphotungstic acid haematoxylin, $\times 245$.

some relationship between cerebral infiltration and the presence of an astrocytic reaction. Fourteen (75\%) of the 19 tumours with a glial reaction contained mitotic figures, as against $45 \%$ in the whole series of tumours. Also all 17 of the infiltrating tumours contained mitoses, whereas only $20(40 \%)$ of the 53 non-infiltrating tumours did so. These findings suggested, but did not prove, a relationship between mitotic figures, infiltration of the brain and an astrocytic response. Possibly all of these merely indicate rapid growth.

The recurrence ratio for the tumours apparently completely removed and showing evidence of cerebral infiltration was $2 / 3(0.66)$ and those definitely not infiltrating the brain was $22 / 5(4 \cdot 4)$. Though the numbers are inadequate, there is an indication of cerebral infiltration being suggestive of recurrence, and certainly 22 of the 24 long survivals showed no evidence of cerebral infiltration by the tumour.

\section{PARTIAL SURGICAL REMOVAL OF THE TUMOUR}

The calculations of the recurrence ratio so far have been only on patients in whom the surgeon felt sure of complete removal of the tumour. This was? in order to minimize the factor of residual tumours as a contribution towards recurrence, and to bringo into focus other factors that might be of imports ance. The recurrence ratio for the 40 patients in whom the surgeon thought he had left tumour behind was $15 / 61(0.93)$ and for the 141 in whom he felt sure of complete removal $85 / 20(4 \cdot 25)$. Thisi indicates that residual tumour is a cause of recur- 3 rence. It is therefore of interest that if the recurrence ratios of the pathological factors just examinedP in the patients with complete removals, are also calculated in those with residual tumours, they still show the same influence towards recurrence N They are effective enough not to be overshadowed or eradicated by the residual tumour and the 
selective irradiation of tumours in which removal is thought to be incomplete. In these patients with residual tumour, the recurrence ratio for the different tumour types was syncytial $2 / 4(0.5)$, transitional 11/7 (1.6) and fibroblastic $3 / 2(1 \cdot 5)$. That for cases with mitotic figures was $3 / 11(0 \cdot 27)$ as against those with no mitotic figures $11 / 5(2 \cdot 2)$. That for cases with focal necrosis was $1 / 6(0 \cdot 16)$ as against those with no focal necrosis $13 / 8(1.62)$. That for cases with cerebral infiltration was $1 / 4(0.25)$ as against those with no cerebral infiltration $5 / 6$ $(0 \cdot 83)$. The numbers in these calculations are small, and like all those in this series do not merit statistical analysis. However, the fact that the same factors point in the same direction, toward recurrence, in cases with and without residual tumour adds credence to their validity. This is summarized in Table 6 which shows all the recurrence ratios in this series.

\section{TABLE 6}

RECURRENCE RATIOS FOR THE FACTORS EXAMINED IN PATIENTS WITH COMPLETE AND INCOMPLETE REMOVAL OF MENINGIOMAS

\begin{tabular}{lcc}
\hline & Complete removal & Incomplete removal \\
\cline { 2 - 3 } All cases & $85 / 20(4 \cdot 25)$ & $15 / 61(0 \cdot 93)$ \\
Syncytial & $13 / 7(1 \cdot 9)$ & $2 / 4(0 \cdot 5)$ \\
Transitional & $41 / 12(3 \cdot 4)$ & $11 / 7(1 \cdot 6)$ \\
Fibroblastic & $20 / 1(20)$ & $3 / 2(1 \cdot 5)$ \\
Mitotic figures & $26 / 14(1 \cdot 85)$ & $3 / 11(0 \cdot 27)$ \\
No mitotic figures & $55 / 6(9 \cdot 1)$ & $11 / 5(2 \cdot 2)$ \\
Focal necrosis & $8 / 8(1 \cdot 0)$ & $1 / 6(0 \cdot 16)$ \\
No focal necrosis & $78 / 12(6 \cdot 5)$ & $13 / 8(1 \cdot 62)$ \\
Cerebral infiltration & $2 / 3(0 \cdot 66)$ & $1 / 4(0 \cdot 25)$ \\
No cerebral infiltration & $22 / 5(4 \cdot 4)$ & $5 / 6(0 \cdot 83)$ \\
\hline
\end{tabular}

CONCLUSION AND SUMMARY

It is concluded as a result of this investigation of the pathology of parasagittal and falcine meningiomas, that the pathologist should take note of the type of the tumour and the presence of mitotic figures, focal necrosis, and cerebral infiltration. The syncytial meningiomas are most likely to recur and the fibroblastic ones least so. Mitotic figures, focal necrosis, and cerebral infiltration are suggestive of recurrence. The numbers of the uncommon angioblastic meningiomas are small in this series, but in spite of their frequently containing mitoses and regions of focal necrosis, they did not recur if completely removed. This should not be taken to be characteristic until larger series are examined.

It is suggested that if a surgeon is satisfied that he has completely removed a meningioma, and if the pathologist finds a fibrous or even a transitional tumour with no mitoses, focal necrosis, or cerebral infiltration, then the patient can be spared postoperative irradiation, the effectiveness of which is dubious anyway.

Incomplete removal of a tumour contributes towards recurrence and the factors of mitoses, etc., mentioned above still influence recurrence in this type of case-that is, residual syncytial tumour containing mitotic figures is more likely to produce clinical recurrence than is a residual fibroblastic tumour with no mitotic figures.

We are grateful to Mr. Wylie McKissock who suggested this investigation and gave us access to the records. We are also grateful to Professor T. Crawford of the Department of Pathology, St. George's Hospital Medical School, for his assistance with the photomicrography and to both for their helpful criticism. We are additionally grateful to Professor W. Blackwood, of the Department of Neuropathology at the National Hospital for Nervous Diseases, Queen Square, and his secretary, Miss J. Silverwood, for their great help in supplying the material from their department. We are also grateful to Mrs. Y. Bosley for her secretarial assistance.

REFERENCES

Courville, C. B. (1945). Pathology of the Central Nervous System. A study based upon a survey of lesions found in a series of 30,000 autopsies. 2nd edn. Pacific Press: Mountain View, California.

Cushing, H. W., and Eis snhardt, L. (1938). The Meningiomas. Thomas: Springfield, Illinois.

Russell, D. S., and Rubinstein, L. J. (1959). Tumours of meninges and of related tissues, pp. 42-62. In Pathology of Tumours of the Nervous System. Edward Arnold: London.

Simpson, D. (1957). The recurrence of intracranial meningiomas after surgical treatment. J. Neurol. Neurosurg. Psychiat., 20, 22-39. 